\title{
Ferroptosis and Brain Injury
}

\author{
Leslie Magtanong Scott J. Dixon \\ Department of Biology, Stanford University, Stanford, CA, USA
}

\section{Keywords \\ Necrosis · Iron · Polyunsaturated fatty acids · PUFAs - GPX4 . Glutathione $\cdot$ Stroke $\cdot$ Ischemia-repurfusion injury}

\begin{abstract}
Ferroptosis is a nonapoptotic form of cell death characterized by the iron-dependent accumulation of toxic lipid reactive oxygen species. Small-molecule screening and subsequent optimization have yielded potent and specific activators and inhibitors of this process. These compounds have been employed to dissect the lethal mechanism and implicate this process in pathological cell death events observed in many tissues, including the brain. Indeed, ferroptosis is emerging as an important mechanism of cell death during stroke, intracerebral hemorrhage, and other acute brain injuries, and may also play a role in certain degenerative brain disorders. Outstanding issues include the practical need to identify molecular markers of ferroptosis that can be used to detect and study this process in vivo, and the more basic problem of understanding the relationship between ferroptosis and other forms of cell death that can be triggered in the brain during injury.

(c) 2019 S. Karger AG, Basel
\end{abstract}

\section{Introduction}

During development and in adulthood, damaged or unwanted cells are eliminated through the activation of different regulated cell death pathways. In many devel- opmental contexts, the caspase-dependent apoptosis pathway executes cell death [1]. However, in other contexts, especially those involving infection or trauma, cell death is executed by nonapoptotic cell death pathways, including necroptosis, pyroptosis, parthanatos, and ferroptosis [2-6]. Some of these pathways have been linked to pathological cell death in the nervous system. For example, necroptosis is associated with axonal degeneration in amyotrophic lateral sclerosis and frontotemporal dementia $[7,8]$, while parthanatos, i.e., poly-(ADPribose)-polymerase-1 (PARP1)-dependent cell death, is linked to neuronal cell death during glutamate-induced excitotoxicity and stroke [9-11]. In this review, we focus on ferroptosis, a nonapoptotic form of cell death first reported in $2012[12,13]$. This process was initially characterized in vitro in cancer cells using synthetic small molecules, but it has since been linked to cell death events observed in vivo following acute injury to the brain and other tissues [14-20].

At the molecular level, ferroptosis is characterized by the iron-dependent accumulation of lipid reactive oxygen species (ROS), which are associated with irreparable lipid damage and membrane permeabilization. Recent studies have defined key enzymes and metabolites that either promote or prevent ferroptosis, and pinpointed chemical modulators of this process [13]. It is important to note that iron-dependent cell death is not a novel concept [21, $22]$. The neurodegeneration and brain injury literature contains many descriptions of pathological cell death events linked to iron and/or oxidative stress [23-30].

\section{KARGER}

(C) 2019 S. Karger AG, Basel 
Fig. 1. a Key metabolites and enzymes (bold text) involved in the ferroptosis pathway. Insets Upper: the regulation of cystine (Cys) import by glutamate (Glu) levels. Lower: additional detail on the regulation of lipid metabolism in ferroptosis. The purple-shaded box highlights the iron-catalyzed, free radical-mediated chain reaction of lipid oxidation. b The table lists key small-molecule modulators of ferroptosis and the associated mechanisms of action.

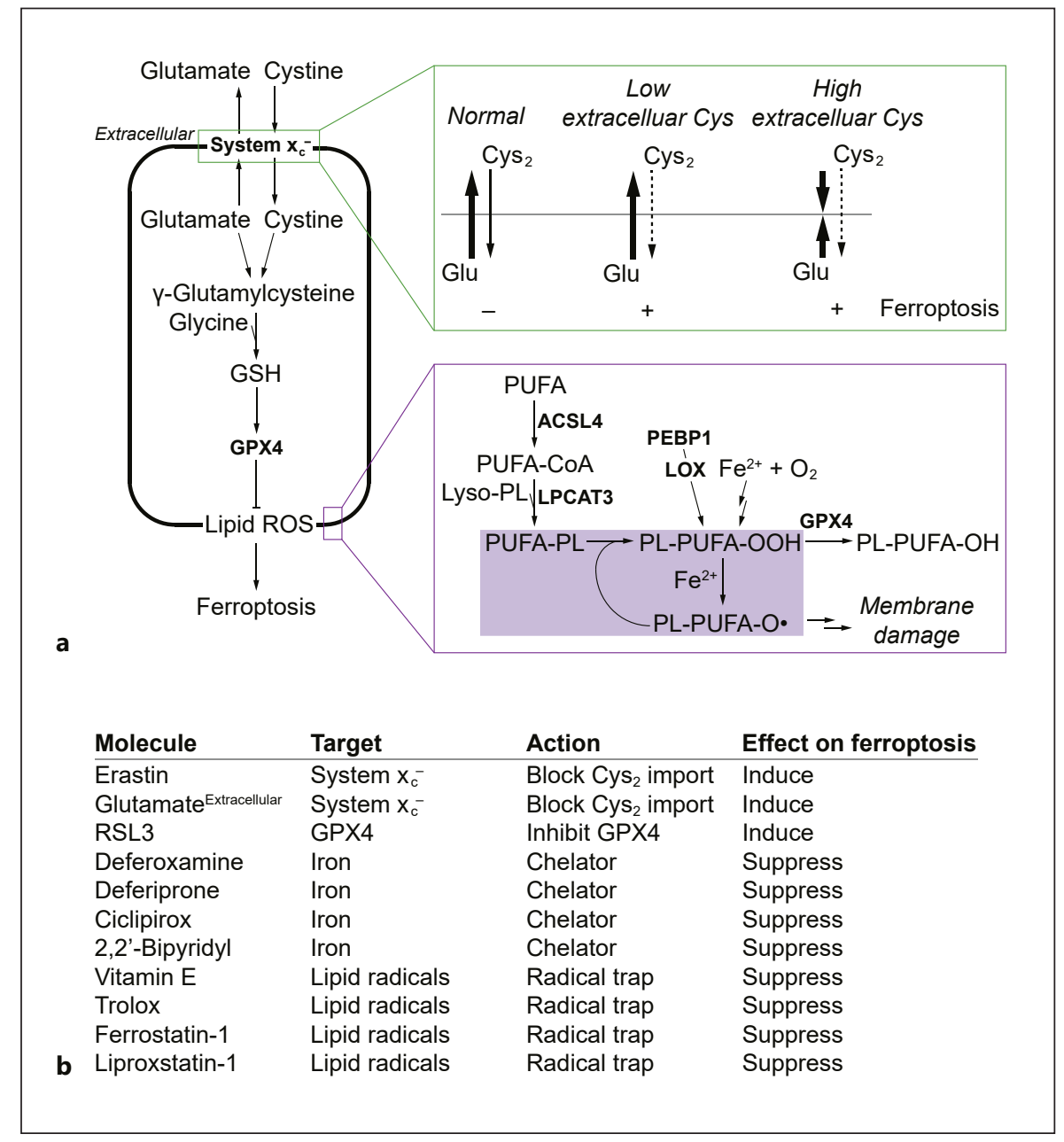

However, much of this work pre-dates the discovery that nonapoptotic cell death (i.e. necrosis) can be a highly regulated process [25]. It is therefore possible that many previous observations linking iron and ROS to brain injury could be explained by the induction of ferroptosis. A better understanding of the ferroptosis pathway and what makes this process unique could lead to new therapies that specifically block with this process in the brain and other tissues. Here, we review the discovery of ferroptosis, the relationship between this process and other forms of oxidative cell death that have been described previously, and recent examples of links between ferroptosis and brain injury.

\section{The Mechanism of Ferroptosis}

Ferroptosis is characterized by the iron-dependent accumulation of toxic lipid ROS (Fig. 1a). In particular, ferroptosis occurs when the oxidation of membrane polyun- saturated fatty acids (PUFAs) is allowed to run out of control due to inactivation of the lipid hydroperoxidase glutathione peroxidase 4 (GPX4) $[18,31,32]$. It is presumed that unrestrained lipid oxidation and lipid ROS formation ultimately result in membrane damage and perforation [26], but the precise chemical details of how this occurs remain unclear. Unlike other forms of cell death, this process is not known to require the transcriptional upregulation or posttranslational modification of any specific cell death effector or pore-forming protein. Indeed, as far as is understood, there is no ferroptosis program latent in the cell and waiting to be activated. Rather, ferroptosis is triggered when disruption of the continuously operating endogenous antioxidant network of the cell results in lipid ROS accumulation to toxic levels [22]. Due to the central role of iron-dependent lipid peroxidation, this process can be inhibited by natural product and synthetic small-molecule iron chelators, such as deferox- 
amine and ciclopirox [12, 27]. Lipophilic antioxidants, including vitamin E, trolox, ferrostatin-1, and liproxstatin-1 are also potent ferroptosis inhibitors (Fig. 1b).

Ferroptosis was initially identified and studied in vitro in human cancer cells treated with the synthetic small molecules erastin and 1S,3R-RSL3 (hereafter RSL3) [28, 29] (Fig. 1b). These molecules were discovered in phenotypic small-molecule screens based on their ability to selectively induce cell death in certain cancer cells. In sensitive cells, treatment with erastin and RSL3 does not cause caspase activation or lead to other biochemical or morphological features of apoptosis (e.g., chromosomal margination) $[27,28]$. Bioenergetically, ferroptosis triggered by erastin occurs without the loss of intracellular adenosine triphosphate (ATP), which distinguishes this process from unregulated necrosis and PARP-dependent cell death [12]. Morphologically, the cell death phenotype induced by these molecules in human cancer cells is clearly distinct from apoptosis, unregulated necrosis (e.g., induced by $\mathrm{H}_{2} \mathrm{O}_{2}$ ), and autophagy, which can contribute to cell death in some contexts [12]. The only notable morphological phenotype of cells undergoing ferroptosis is an alteration in mitochondrial morphology, including smaller size and disorganized cristae $[12,18,27]$. The cause of these changes and whether they are functionally important to the execution of ferroptosis are important unresolved questions. Nonetheless, these changes may be sufficiently characteristic to identify ferroptotic cells in vivo [30, 31].

The protein targets of erastin, RSL3, and related molecules act to restrain ferroptosis. Erastin is a potent and specific inhibitor of the system $\mathrm{x}_{c}{ }^{-}$cystine/glutamate antiporter, a heterodimer composed of the SLC3A2 (4H2hc, CD98) regulatory subunit and the SLC7A11 (xCT) 12-pass transmembrane protein [12, 32, 33]. System $\mathrm{x}_{\mathrm{c}}{ }^{-}$imports extracellular cystine in for exchange for intracellular glutamate. This transport cycle is ATP-independent, being driven by the high concentration of intracellular glutamate. This renders system $\mathrm{x}_{\mathrm{c}}{ }^{-}$sensitive to inhibition by high concentrations of extracellular glutamate, as can occur in a variety of brain injury contexts [34] (Fig. 1a). Once transported into the cell, cystine is reduced to cysteine, which is used to synthesize proteins and the nonribosomal tripeptide antioxidant (reduced) glutathione (GSH). Of the 3 amino acids found in GSH, cysteine is typically the least abundant, and therefore considered rate-limiting for de novo GSH synthesis. Cysteine can be synthesized in some cells from methionine, via the transsulfuration pathway. However, in many other cells, cystine import appears to be essential to maintain cysteine and GSH levels and prevent ferroptosis, at least in vitro.

RSL3 and related molecules covalently bind to and inhibit the function of the GSH-dependent selenoenzyme GPX4 [35, 36]. Loss of GPX4 function either directly (e.g., due to covalent inhibition) or indirectly (e.g., due to GSH depletion) is currently thought to be the key event leading to the onset of ferroptosis. This is due to the central role of GPX4 in the prevention of lethal lipid peroxidation. Relative to saturated fatty acids (SFAs) and monounsaturated fatty acids (MUFAs), the bis-allylic carbons of PUFAs are highly susceptible to oxidation. GPX4 uses GSH as a cofactor to reduce PUFA lipid peroxides (L-OOH) to lipid alcohols (L-OH) (Fig. 1a). The catalytic cycle of GPX4 consumes 2 GSH molecules which are converted to GSSG. GSSG can be recycled back to GSH by GSH reductase in an NADPH-dependent manner. Normally, a balance is maintained between $\mathrm{L}-\mathrm{OOH}$ formation and L$\mathrm{OOH}$ reduction to L-OH by GPX4. Lipid hydroperoxides $(\mathrm{L}-\mathrm{OOH})$ can be oxidized by $\mathrm{Fe}^{2+}$ to yield highly reactive alkoxy radicals $(\mathrm{L}-\mathrm{O} \bullet)$. These radicals can directly damage adjacent PUFAs through free radical-mediated chain reactions (Fig. 1a, purple-shaded area) [37]. When GPX4 is absent or inactivated, $\mathrm{L}-\mathrm{OOH}$ can accumulate to higher levels than normal, resulting in a greater production of $\mathrm{L}-\mathrm{O} \bullet$, presumably leading to catastrophic membrane damage. As predicted from this model, lipidomic studies show that cells treated with erastin are depleted for various PUFAs, whereas the levels of these species are maintained in cells cotreated with erastin and the radical-trapping antioxidant ferrostatin-1, despite the effective inhibition of system $\mathrm{x}_{\mathrm{c}}{ }^{-}$and the depletion of intracellular GSH [38]. These findings place lipid oxidation downstream of GSH depletion and/or GPX4 inactivation in the ferroptotic cascade.

Various PUFA-containing phospholipids (PLs) are oxygenated during ferroptosis, with PUFA-containing phosphatidylethanolamines (PEs) being highly oxidized following GPX4 inactivation in mouse fibroblasts [39, 40]. The PE C20:4 (arachidonoyl) and C22:5 (adrenoyl) fatty acyl chains, in particular, are doubly or triply oxygenated following Gpx4 inactivation [39]. The incorporation of these PUFAs into PLs, and thus sensitivity to ferroptosis, is attenuated by the disruption of specific lipidmetabolic enzymes required for the metabolism of PUFAs within the cell. To date, genetic screens in cancer cells and mouse embryonic fibroblasts (MEFs) have identified 2 lipid-metabolic enzymes that are required to execute ferroptosis, at least in certain nonneuronal cells [39-42]. Acyl-CoA synthetase long-chain family member 4 (ACSL4) 
preferentially activates PUFA free fatty acids to PUFACoAs, and this activation is required for their incorporation into PLs. Lysophosphatidylcholine acyltransferase 3 (LPCAT3) is an enzyme that inserts acyl-CoA molecules into lysophosphatidylcholine to generate phosphatidylcholine. LPCAT3 preferentially acts on PUFA-CoAs, including arachidonoyl-CoA. Together, these 2 enzymes help define a lipid metabolic pathway essential for PUFA insertion into membrane PLs and sensitivity to ferroptosis. Recent evidence suggests that it is the PUFA-containing PLs found at the plasma membrane whose oxidation is necessary for ferroptosis, and that the relative ratio of plasma membrane PLs acylated with MUFAs (e.g., oleic acid) versus PUFAs (e.g., arachidonic acid) helps determine cellular ferroptosis sensitivity (Magtanong et al. [128]). It is possible that cells dynamically modulate the "oxidizability" of the plasma membrane by manipulating the relative levels of PUFAs versus MUFAs that are incorporated into this structure.

The requirement for iron is a defining feature of ferroptosis. Both ferric and ferrous iron chelators (deferoxamine and ciclopirox, respectively), but not chelators of other divalent metals, are effective inhibitors of ferroptotic cell death [13]. Iron is carried in the bloodstream by the glycoprotein transferrin. Depletion of transferrin from cell culture medium can block ferroptotic cell death of MEFs, and resupplementation with holotransferrin (i.e., iron-loaded) but not apotransferrin (i.e., iron-free) restores ferroptosis in this system [15]. In cancer cells, genetic silencing of key iron metabolic genes including $I R E B 2$ and TFRC (encoding the transferrin receptor) reduce iron uptake and ferroptosis sensitivity, while perturbation of mitochondrial iron/sulfur cluster biogenesis can increase ferroptosis sensitivity $[12,15,29,43]$. How iron impacts lipid oxidation to promote ferroptosis is not yet completely understood. Intracellular $\mathrm{Fe}^{2+}$ can promote the formation of hydroxyl and alkoxyl radicals that can initiate or help propagate lipid ROS production. Additionally, the function of the lipid-oxidizing lipoxygenase (LOX) family of enzymes is iron-dependent, and genetic silencing of LOX enzyme expression can block ferroptosis in some models, as anticipated from early studies of glutamate toxicity $[44,45]$. In some contexts, LOX function and the oxidation of specific PUFA-containing PEs is orchestrated by 15 -LOX binding to phosphatidylethanolamine-binding protein 1 (PEBP1) (Fig. 1a). PEBP1 binds LOX and orients PE-PUFAs at the membrane, facilitating lipid oxidation [20]. Whether there is a universal requirement for LOX activity, however, remains unclear. Joint deletion or silencing of Alox15 and
Alox5 does not impair ferroptosis induced by Gpx4 deletion in mice [18], and in at least 1 animal model of brain injury, the Alox5 enzyme appears most important [46]. Moreover, some cultured cells completely lacking LOX enzyme expression execute ferroptosis normally [47]. Thus, the role of individual LOX enzymes in ferroptosis may be species- and/or tissue-specific. Importantly, many commonly employed putative LOX inhibitors (e.g., nordihydroguaiaretic acid [NDGA] and zileuton) can act as direct radical-trapping antioxidants $[47,48]$, which could account for their ability to inhibit ferroptosis and related forms of oxidative cell death, apart from LOX inhibition.

\section{Ferroptosis, Oxidative Glutamate Toxicity, and}

Oxytosis: A Common Process?

Given the mechanistic details elucidated above, it is likely that ferroptosis is related or identical to oxidative cell death phenotypes first observed as early as 1977 in fibroblasts and cultured brain cell lines deprived of cystine or exposed to high levels of extracellular glutamate $[45,49-55]$. This process was termed oxidative glutamate toxicity, and subsequently oxytosis, to distinguish it from apoptosis $[45,56]$. The central role of system $\mathrm{x}_{\mathrm{c}}{ }^{-}$inhibition, GSH depletion, and iron-dependent lipid ROS accumulation is broadly consistent in studies of ferroptosis and oxytosis, and it may represent the core of 1 common lethal process [57-59]. However, glutamate-induced oxytosis in neuronal-like cells displays certain differences when compared to ferroptosis in cancer cells, so the actual degree of overlap between oxytosis and ferroptosis still remains somewhat murky $[57,58]$. Reconciling these differences has not been straightforward, as most mechanistic studies on ferroptosis have been carried out in cancer cells and fibroblasts, while most studies on oxytosis have been carried out in neurons or neuronal-like cells, especially the HT-22 cell line.

In human cancer cells, the accumulation of lipid ROS appears necessary and sufficient for membrane permeabilization and the induction of ferroptosis. Moreover, $\mathrm{Ca}^{2+}$ influx and mitochondrial function are not necessary for the execution of ferroptosis [12, 59], although they may contribute to the process (e.g., [81, 82]). By contrast, in neuronal cell models (e.g., the HT-22 cell line), additional molecular events are required downstream of lipid ROS accumulation for the execution of cell death, including $\mathrm{Ca}^{2+}$ influx into the cell via ORAI calcium releaseactivated calcium modulator 1 (ORAI1), BH3-interacting domain death agonist (BID) translocation to the mitochondria, mitochondrial ROS production, and the release of apoptosis-inducing factor (AIF) from mitochondria 
[50, 60-66]. Whether the execution of oxytosis requires the recently identified AIF-binding partner, the nuclease macrophage migration inhibitory factor (MIF), is unclear [11]. It is possible that these downstream events dependent on $\mathrm{Ca}^{2+}$, mitochondria, and AIF are specific to neurons, and represent a module that is "bolted on" to the core GSH-GPX4-lipid-ROS ferroptotic pathway in these cells. Indeed, AIF appears dispensable for cell death in fibroblasts, T cells, and B cells, suggesting that the role of this enzyme could be tissue-specific [67]. From a therapeutic standpoint, it might not matter. Given that lipid ROS accumulation lies in the shared portion of the pathway common to both mechanisms, targeting this molecular event should be sufficient to block ferroptosis/oxytosis in all cases.

\section{Ferroptosis and Development}

Whether ferroptosis is engaged during development to eliminate unwanted cells in a programmed manner is unknown. With a few notable exceptions (e.g., cornification of skin epithelia), nonapoptotic cell death pathways do not appear to play a primary role in most developmental contexts, although they can serve an important backup function $[3,68]$. It is therefore perhaps not surprising that, to date, there is little evidence that ferroptosis is required for normal development. In the mouse autopod, a decrease in Gpx4 activity correlates with increased interdigital cell death, a process that can occur in a caspaseindependent manner, but whether this is a primary means of eliminating these cells or a backup mechanism is not clear $[60,61]$. In mice lacking the $\mathrm{E} 3$ ligase gene, mouse double minute $2(M d m 2)$, and where the endogenous Trp53 gene has been mutated to encode a variant with 3 lysines in place of arginine, treatment with the ferroptosis inhibitor ferrostatin-1 can improve embryonic development, consistent with the possibility that p53 may promote cell death in the developing embryo, in part via ferroptosis [62]. However, these studies do not suggest a primary role for ferroptosis in the elimination of a defined cell population. Rather, these findings, and those described below, are more consistent with the model that GPX4 and other enzymes in the ferroptosis pathway are simply essential for cell viability.

GPX4 is expressed in the brain of developing animals. In rats, Gpx4 mRNA and protein are detected at various stages of cerebral development, with the hippocampus being one structure that maintains high Gpx4 expression into adulthood [63]. In the adult rat brain, dentate granule cells and hippocampal cornu ammonis pyramidal neurons express Gpx4. The pyramidal neurons in the frontal and entorhinal cortex exhibit strong Gpx4 expression [63]. In an in vitro model of embryogenesis, 2 of the $3 \mathrm{Gpx} 4$ isoforms (mitochondrial, m-Gpx4; and nuclear, $\mathrm{n}$-Gpx4) were shown to promote normal development. Injection of small-interfering (si)RNA against n-Gpx4 led to a defect in development of the left atrium of the heart [64]. Injection of $\mathrm{m}-\mathrm{Gp} \times 4$ siRNA resulted in drastic defects of rhombomeres 5 and 6 of the developing hindbrain, and the neuroepithelium between these 2 rhombomeres was smaller than normal [64]. The importance of Gpx4 activity during embryonic brain development was also demonstrated by studying the posttranscriptional regulation of m-Gpx4 mRNA. Guanine-rich sequencebinding factor 1 ( $\mathrm{Grsfl} 1$ ) is an RNA-binding protein that binds to a 14-base pair G-rich element sequence [65]. Grsf1 binds to a specific region in the $5^{\prime}$ end of $m-G p \times 4$ mRNA, and mRNA of both genes was detected in the embryonic brain [66]. siRNA silencing of Grsf1 results in decreased m-Gpx4 expression, higher levels of isoprostanes (indicative of increased lipid peroxidation) and cell death, and defects in midbrain and hindbrain development [66]. These findings are corroborated by the re-

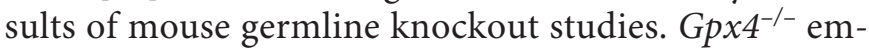
bryos die at embryonic day 7.5 (E7.5), exhibiting failed gastrulation and very little germ layer differentiation [69]. Whole-body deletion of Gpx4 deletion in adults is also lethal due to kidney failure, while adult tissue-specific deletion of this gene can result in the loss of neuronal and skin cell viability $[18,70,71]$. Intriguingly, introduction of a mutation causing replacement of the Gpx 4 active site selenocystine with a less-reactive cysteine residue results in enzyme inactivation due to irreversible hyperoxidation; however, this is compatible with normal embryonic development, and animals ultimately die only around postnatal day 15 due to the loss of parvalbumin-positive GABAergic interneurons that leads to fatal seizures [19]. Thus, only minimal Gpx4 activity appears to be required for normal embryonic development, and this enzyme plays a more important role in postnatal cell survival.

Unlike GPX4, deletion of the system $\mathrm{x}_{\mathrm{c}}{ }^{-}$transport subunit Slc7a11 in mice does not impair development and has only mild effects on homeostasis in adults [72-74]. One possibility is that the requirement for system $\mathrm{x}_{\mathrm{c}}{ }^{-}$ function to prevent ferroptosis only manifests under tissue culture conditions (e.g., high oxygen) [72]. Another possibility is that deletion of $S l c 7 a 11$ results in metabolic compensation that enables these mutant cells to maintain intracellular cysteine and GSH levels by alternative means. For example, loss of system $\mathrm{x}_{\mathrm{c}}{ }^{-}$function could 
lead to upregulation of the transsulfuration pathway, which generates cysteine from methionine. This possibility would be consistent with recent knockout studies that have confirmed extensive plasticity between redox pathways in development [75]. The expression of antioxidant enzymes including GPX1 and superoxide dismutase 1 (SOD1) can vary over time during development, altering sensitivity to brain injury $[76,77]$. Whether the expression levels of GPX4, SLC7A11, and other key regulators of ferroptosis per se change over time during development and aging, or in response to slow-onset neurological diseases (e.g., [78]), in a manner that influences ferroptosis sensitivity in different tissues, requires further investigation.

\section{Ferroptosis and Brain Injury}

The first report describing ferroptosis linked this process to acute brain injury, establishing the concept that these 2 processes may be related [12]. Exposure of dissected rat organotypic hippocampal slices to a high concentration of extracellular glutamate induced cell death that could be partially (around 50\%) suppressed by cotreatment with ferrostatin-1 or ciclopirox, consistent with the induction of ferroptosis. Subsequently, improved ferrostatin-1 analogs were tested in an in vitro model of periventricular leukomalacia. Previous studies showed that periventricular white matter injury was associated with elevated levels of lipid peroxidation, and that cell death was exacerbated by iron supplementation or de novo GSH synthesis inhibition and suppressed by $\mathrm{N}$-acetylcysteine [79-81]; this appeared reminiscent of ferroptosis. Indeed, ferrostatin-1 and its improved analogs could block cell death in this model of oligodendrocyte death [38]. Moreover, the lethality of extracellular glutamate towards cultured oligodendrocytes is suppressed by ferrostatin-1, liproxstatin-1, iron chelators, and other small molecules known to inhibit ferroptosis [82]. Cell death under these conditions is not blocked by the pancaspase inhibitor Z-VAD-fmk or the necroptosis inhibitor necrostatin-1s, and it does not lead to the formation of necrosome complex, further ruling out the involvement of apoptosis and necroptosis, respectively, in this process.

Emerging evidence implicates ferroptosis as a bona fide mechanism of acute pathological brain cell death in vivo. This was investigated by Tuo et al. [83] in the context of unilateral transient middle cerebral artery occlusion (MCAO). These investigators focused specifically on the role of tau, amyloid precursor protein (App), and the ferrous iron exporter ferroportin (Fpn) in iron-handling, and how this impacted tissue injury and behavior. In rat and mouse models, MCAO resulted in elevated iron levels in the lesioned hemisphere that correlated with a reduction in iron export. It was hypothesized that this iron accumulation could sensitize to, or perhaps induce, ferroptosis. Consistent with such a model, liproxstatin-1 or ferrostatin-1 treatment limited the infarct size and reduced the behavioral losses occasioned by $\mathrm{MCAO}$, even when administered $6 \mathrm{~h}$ after reperfusion. The authors proposed that, through an unknown mechanism, ischemia results in reduced tau protein. This, in turn, leads to the inhibition of App-regulated, Fpn-dependent iron export. Loss of iron export results in intracellular iron accumulation and increased susceptibility to and/or the induction of ferroptosis. Another means of increasing the local concentration of iron at the site of injury could involve an influx of holotransferrin from leaking blood vessels near the damaged area [84]. Interestingly, increasing the levels of apotransferrin in the bloodstream (to lower the percentage transferrin saturation) decreased brain injury and improve neurological outcomes, likely associated with reduced neuronal lipid peroxidation and cell death. Other studies confirm the efficacy of ferroptosis inhibitors in the inhibition of MCAO-induced brain damage but suggest that additional nonapoptotic processes could also be active here [85].

Another process in which ferroptosis has been implicated is brain injury following intracerebral hemorrhage, which results from blood vessel rupture and the leakage of blood into the brain. During this process, iron from hemoglobin found in the blood can be released and cause neuronal damage by enhancing ROS formation [30]. Ferrostatin-1 or liproxstatin-1 can inhibit cell death in the hippocampal region of brain-slice cultures treated directly with hemoglobin or free iron. Mechanistically, exposure to hemoglobin-bound iron leads to GSH depletion and GPX4 inactivation in these hippocampal cells. In an in vivo model of collagenase-induced vessel damage, direct injection of ferroptosis inhibitors at or distal to the site of injury reduced the number of damaged cells and the size of the injury, improving the subsequent neurological function of the animals [30]. Protection against intracerebral hemorrhage-associated cell death is also conferred by the cell-permeable cysteine analog, $\mathrm{N}$-acetyl-cysteine (NAC) [46]. Intracellular cysteine can contribute to the production of GSH, and these results therefore indicate that ferroptosis following intracerebral 
hemorrhage results from a defect in GSH synthesis (e.g., a loss of cystine uptake).

In a rat model of intracerebral hemorrhage, partial protection against cell death is also conferred by overexpression of Gpx4, whose levels in the brain drop in the damaged area in the immediate aftermath of the injury [86]. Brain injuries involve a complex interplay between different cell types, and, interestingly, bone marrow-derived polymorphonuclear neutrophils (PMNs) that infiltrate the site of damage following intracerebral hemorrhage can be stimulated by interleukin 27 to release lactotransferrin, an iron-binding molecule that can clear free iron from the damaged area, leading to decreased levels of 4-hydroxynonenal (4-HNE), a lipid peroxidation breakdown product, and improved neurological outcomes [87]. Speculatively, but in line with other recent results, this beneficial effect could involve a reduction in the degree of ferroptosis within the damaged area.

Ferroptosis has also been linked to traumatic brain injury. In a rat controlled cortical-impact model, elevated levels of oxidized PE lipid species could be detected $1 \mathrm{~h}$ after injury, consistent with the loss of GPX4 function [20]. In a mouse controlled cortical-impact model of traumatic brain injury, a loss of iron-positive cells and morphological evidence consistent with ferroptosis (i.e., shrunken mitochondria) was observed at or near the injury site [31]. Moreover, cell death and the associated behavioral changes were reduced by cotreatment with ferrostatin-1, administered at the time of injury directly into the cerebral ventricles. Other studies of brain compression injury demonstrate an increase in ROS accumulation and cell death that can, likewise, be partially suppressed by direct administration of reduced GSH [88]. This is consistent with other ex vivo data that ferroptosis and other cell death processes may act in parallel [12], especially following traumatic brain injury. Indeed, it is possible that previous studies linking brain injury to both apoptosis and nonapoptotic (i.e., necrotic) processes can be rationalized by assuming that each different process (e.g., apoptosis, parthanatos, and ferroptosis) contributes to the death of different cells, depending on the type of stress experienced by a particular region of the brain [89-91].

\section{Ferroptosis and Acute Brain Injury: Reinterpretation of Early Studies}

With our new understanding of ferroptosis, it is possible to revisit results obtained previously that further support the notion of ferroptosis as a bona fide mecha- nism of neuronal cell death occurring after acute brain injury in both the developing and adult brain. For example, free iron accumulates in the ipsilateral hypoxic-ischemic neonatal rat cortex as early as $4 \mathrm{~h}$ postinjury [92], and the iron chelator deferoxamine protects from neonatal brain damage sustained after hypoxia-ischemia [93, 94]. Children diagnosed with traumatic brain injury have increased levels of $\mathrm{F}_{2}$-isoprostane, a biomarker of lipid peroxidation, and decreased levels of the antiferroptotic metabolite GSH [95]. These features are highly suggestive of ferroptosis, but the involvement of this process per se remains to be confirmed in this and other models that use highly potent and specific inhibitors such as ferrostatin-1 or liproxstatin-1.

New understanding of small molecule inhibitor effects may also provide evidence for the importance of ferroptosis in brain injury. One of the first small-molecule MEK1/2 inhibitors, U0126, reduces neuronal cell death and brain atrophy in gerbils following MCAOinduced injury [96], as well as glutamate-induced toxicity in HT-22 cells in vitro [97]. These effects were attributed to the ability of this compound to block MEK1/2 activity and MAPK pathway activity. Later, U0126 was identified as a potent inhibitor of ferroptosis in cancer cell-based models, and likewise attributed to the inhibition of MEK $1 / 2$ activity [27]. However, it is now clear that U0126 can act as a free radical scavenger and that its ability to block ferroptosis is not necessarily linked to the inhibition of MEK1/2 activity [15]. By inference, some of the protective effects of U0126 observed previously in brain injury studies may be attributable to the inhibition of ferroptosis via an off-target antioxidant mechanism.

A similar example is provided by necrostatin-1, a potent inhibitor of RIPK1 kinase activity and the RIPK1dependent nonapoptotic process of necroptosis. Necrostatin-1 was shown to reduce infarct size in the brain in an MCAO model of stroke in mice [25]. Problematically, at high concentrations necrostatin-1 can inhibit ferroptosis through an unknown mechanism that is independent of RIPK1 [18]. It has been observed that necrostatin-1 potently suppresses glutamate-induced ROS accumulation and cell death in HT-22 cells, but only when used at concentrations far above what is needed to inhibit RIPK1 activity [98, 99]. Moreover, studies that have directly compared necrostatin-1 to an improved analog, necrostatin-1s, have shown that only the parent compound possesses the ability to suppress cell death under ferroptosis-inducing conditions in brain cells [82]. Thus, it is possible that some of the pro- 
tective effects of the original parent compound observed previously (e.g., $[25,100])$ may be attributed to the inhibition of ferroptosis. Likewise, as noted above, many putative small-molecule lipoxygenase inhibitors prevent ferroptosis by acting directly as radical-trapping antioxidants, rather than through effects on LOX enzyme function, at least in vitro [47]. The above considerations do not invalidate the previous results; MAPK pathway activity, RIPK1-dependent necroptosis, and LOX enzyme activity could all contribute to neuronal injury in vivo. However, the potential contribution of these processes to ferroptosis must also be considered in the light of new information about how these inhibitors can function as antioxidants.

\section{Ferroptosis and Degenerative Brain Injury}

In addition to acute brain injury, ferroptosis is being linked to the cell death observed in a number of degenerative conditions. There are well-established links between iron and neurodegeneration [101, 102], which likely make these conditions fertile ground in the search for the occurrence of ferroptosis in vivo. For example, overexpression of a pathogenic Huntington (Htt) protein fragment in cultured rat corticostriatal brain slices can trigger cell death that is prevented by cotreatment with ferrostatin-1 [38]. Likewise, dopaminergic cell death in vitro and in vivo caused by exposure to the neurotoxin, 1-methyl-4-phenylpyridinium (MPP+), which produces a Parkinson's-like phenotype, is blocked by ferrostatin- 1 and the iron chelator deferiprone $[78,103]$. Links between ferroptosis and Alzheimer's disease may also exist, given the known associations between this disease and increased iron levels, and the protective effects of certain molecules that can act as antioxidants (e.g., lipoic acid) against disease progression [104, 105]. However, definitive evidence linking neurodegeneration to ferroptosis in long-term animal model studies is currently lacking.

\section{Frontiers in the Investigation of Ferroptosis and Brain Injury}

The vast majority of studies characterizing the biochemical mechanisms of ferroptosis have been performed in cultured cancer cells or MEFs. Even here, basic questions concerning the biochemical regulation of ferroptosis remain. For example, in addition to the key role of GPX4, a second endogenous antioxidant, the metabolite coenzyme $\mathrm{Q}_{10}$, is now thought to be required to suppress lipid ROS accumulation and the onset of ferroptosis [16, 36]. Does coenzyme $\mathrm{Q}_{10}$ act in parallel to GPX4, or are these functions linked in some way? Intriguingly, administration of coenzyme $\mathrm{Q}_{10}$ markedly improved outcomes in a rat MCAO model of stroke [106]. This protective effect was attributed to the inhibition of apoptosis, but it could be worth considering whether this metabolite prevents brain injury by inhibiting ferroptosis. Two related areas where greater mechanistic clarity is required concern the role of different LOX enzymes in promoting ferroptosis and the specific oxidized lipid species that are generated by LOX enzymes and other processes during ferroptosis. There is evidence from cancer cell models that not all ferroptotic triggers cause the same type of oxidative lipid damage [44], and it might be that different cells respond to the same proferroptotic stimulus with unique patterns of lipid oxidation. How different endogenous (e.g., GPX4 and coenzyme $\mathrm{Q}_{10}$ ) and exogenous (e.g., ferrostatin-1 and liproxstatin-1) inhibitors interfere with the production and composition of different oxidized lipid species in brain tissues is also unknown. For all studies conducted using cultured cells, it will also be important to consider whether the composition of the growth medium itself can alter ferroptosis sensitivity [107].

Animal studies investigating the link between ferroptosis and brain injury typically employ inhibitors (e.g., ferrostatins) to block cell death, but have not yet elucidated the specific signals within the complex environment of the brain that actually cause the induction of ferroptosis; in many cases, even the specific class of brain cells that is spared by ferroptosis inhibitors remains unknown. Thus, a fundamental area of future investigation concerns the biochemical regulation of the ferroptotic process in brain cells. It is possible that ferroptosis will exhibit greater regulatory complexity in brain cells or interact with other brain-specific cell death pathways in a way that would not be anticipated from results obtained in cancer cell studies of this process. Studies of the related process of oxytosis already suggest that, in neurons, ferroptosis may exhibit greater regulatory complexity (as described above). However, other complexities are likely to exist due to the unique structure and function of brain cells. NMDA receptor stimulation can induce a molecular cascade that leads to increased neuronal iron uptake via the transferrin receptor and the divalent metal transporter 1 (DMT1), and iron chelation attenuates NMDAinduced excitotoxic neuronal death [108-110]. Could NMDA receptor stimulation-dependent iron uptake sensitize neurons to ferroptosis, or could an increased iron 
uptake itself even be sufficient to trigger ferroptosis in parallel to classic $\mathrm{Ca}^{2+}$-mediated excitotoxicity? If so, this could provoke a re-examination of existing studies of NMDA-receptor mediated neuronal damage, to ascertain whether this involves the induction of ferroptosis. Similarly, links between oxytosis and PARP1-mediated parthanatos may also exist. The execution of both oxytosis and parthanatos is reported to involve the translocation of AIF from mitochondria to the nucleus to trigger DNA degradation and cell death. This implies that, at least in certain brain cells, ferroptosis/oxytosis and parthanatos may converge at the level of AIF, leading to the induction of cell death through a common terminal effector. If so, therapies that target this particular step (i.e., AIF translocation or function) may prove especially useful at blocking pathological brain cell death in cases where both processes are implicated, such as stroke [9-11]. Whether ferroptosis and parthanatos may act in parallel, or in different cell types in response to different configurations of gene expression and ischemia-reperfusion exposure, is an interesting question for future studies.

Another area where progress is required is in the identification of useful molecular markers of ferroptosis that can be employed in vivo or in ex vivo samples. Studies of apoptosis have long benefitted from the ability to detect cleaved caspase- 3 or cleaved PARP as biochemical markers of this process in tissue sections or lysates from damaged brain [111]. Likewise, detection of phosphorylated mixed-lineage kinase domain-like (MLKL) can be used as a molecular marker for execution of necroptosis in various pathological samples [7, 112]. Analogous protein-based molecular markers do not exist for ferroptosis. Fundamentally, this may reflect the fact that the execution of ferroptosis does not appear to require the upregulation or posttranslational modification of any one particular protein that could be the target of a specific antibody, or the surface exposure of a new antigen that could be detected by a specific reagent (e.g., Annexin V), although further investigation is required. To date, the best available markers of ferroptosis are those based on altered gene expressions that occur as a result of ferroptosis. Cysteine deprivation downstream of system $\mathrm{x}_{\mathrm{c}}{ }^{-}$inhibition is sensed by the canonical GCN2/ATF4 pathway, leading to transcriptional upregulation of a battery of ATF4 target genes, including CHAC1 [32]. However, CHAC1 mRNA upregulation is not universal during ferroptosis (e.g., it is not upregulated by direct GPX4 inhibition). Moreover, CHAC1 upregulation is not linked to the execution of ferroptosis in the same way that PARP cleavage or MLKL phos- phorylation are uniquely and mechanistically linked to the execution of apoptosis and necroptosis, respectively. Indeed, CHAC1 levels are increased by diverse ER stresses that cause ATF4 activation, including the inhibition of glycosylation, membrane vesicle transport, and intracellular calcium handling $[113,114]$. An alternative gene expression marker of ferroptosis, PTGES2, may be a broader indicator of the induction of ferroptosis whose expression is increased downstream of either system $\mathrm{x}_{\mathrm{c}}{ }^{-}$ blockade or direct GPX4 inhibition [35]. However, it is unclear whether PTGES2 is specific for ferroptosis or whether this marker would be useful in the context of brain injury.

The optimal molecular marker of ferroptosis in vivo would be linked to the unique features of this cell death program. The best candidate in this connection may be increased levels of PUFA oxidation and membrane lipid ROS. PL oxidation can be detected in brain samples following injury [115], and methods have been developed to perform similar oxylipidomic analyses of cultured cells and animal tissues undergoing ferroptosis due to Gpx4 inactivation or in response to certain injuries (e.g., controlled cortical impact) [20,39]. It is possible that these methods could be applied to samples obtained from the brain under other pathological conditions and examined for patterns of PL oxidation that are known to be characteristic of ferroptosis. Whether ferroptosis results in a unique signature of PL oxidation has not been determined. Furthermore, associating the pattern of lipid oxidation to a specific cell type is currently beyond the technical reach of this method. A final practical consideration is that oxylipidomic methods themselves are highly technical and likely not practical for most investigators to implement independently or routinely.

Another approach is to examine the accumulation of lipid ROS directly. This could potentially be performed in live samples using the probe C11 BODIPY $581 / 591$ (e.g., using a 2-photon microscope or intravital microscopy), or via the administration of a less specific ROS-reactive probe such as Amplex Red [88, 116]. In fixed tissues or lysates, it may be possible to detect 4-HNE or other lipid breakdown products that can modify intracellular proteins to generate a suitable epitope $[37,54,117,118]$, but it is not known if these products are formed during ferroptosis in vivo, and, even if they are, whether they are specific for this process. New chemical probes that report on oxidative damage and are suitable for use in fixed tissues are urgently needed. Currently, functional inhibitor studies demonstrating that a cell death event is blocked by cotreatment with a 
ferroptosis-specific inhibitor are the sole route to confirming the existence of a ferroptotic phenotype in vivo [119].

A final area where progress is required concerns the development of small-molecule ferroptosis inhibitors suitable for advancement to human clinical trials. Ferrostatin-1 is not considered ideal for most in vivo and ex vivo studies due to its poor stability. This has not prevented some investigators from having success with this molecule, both in ex vivo slices and when injecting directly at or relatively near the site of brain damage (e.g., intranasally) $[12,30,38,83]$. Nonetheless, improved analogs of ferrostatin-1 that exhibit better metabolic stability in vivo have demonstrated utility in blocking pathological cell death in animal models of kidney disease, when administered systemically [14, 38]. Whether these molecules or other derivatives of the ferrostatin core scaffold effectively penetrate the blood-brain barrier and/or will be suitable for development into true clinical candidates for use in humans, is presently unclear, despite the promising results of animal studies $[14,120]$. A second distinct class of radical-trapping antioxidant inhibitor is liproxstatin-1. Like improved ferrostatin analogs, liproxstatin-1 has demonstrated antiferroptotic activity in vivo in several animal models $[18,30]$ and, like ferrostatin- 1 , it is widely available commercially. Again, however, like the ferrostatin series, it is not clear whether liproxstatin-1 can or will be developed further for human use.

A more speculative therapeutic direction could be to directly modulate lipid metabolism, with the aim of preventing lipid ROS accumulation in the first place. Small molecules capable of inhibiting ACSL4, an enzyme required for the activation of PUFAs that are subsequently oxidized during ferroptosis, include the natural product triacsin $\mathrm{C}$ and synthetic thiazolidinediones such as rosiglitazone [121]. It is predicted that these would reduce the PUFA load and therefore the "oxidizability" of membrane PLs. Additional inhibitors of ACSL4 could be sought. Alternatively, a potentially distinct approach was established by experiments showing that certain dietary MUFAs such as oleic acid can suppress ferroptosis, at least in cancer cell models in vitro (Magtanong et al. [128]). Given that time would be required for exogenous MUFAs to alter membrane lipid composition, it is unclear whether MUFA treatment would ever be useful in the setting of acute injury. Intriguingly, however, increased MUFA intake may reduce the risk of hemorrhagic but not ischemic stroke [122].

In this review, we have outlined our understanding of the ferroptosis pathway, derived mostly from studies con- ducted on nonneuronal model systems, and describe the contexts in which these may be most applicable to brain injury. Ferroptosis may also contribute to pathological cell death in neurodegenerative processes such as Huntington's disease, Parkinson's disease, and, potentially, degenerative brain conditions characterized by elevated levels of iron $[38,78,123,124]$. How ferroptosis is triggered at the molecular level in different acute and chronic neurodegenerative disorders, and whether the regulation of ferroptosis might differ according to brain cell type, age, or other factors, remains to be elucidated. To answer this question, it will be essential to develop ways to identify the individual cells that undergo ferroptosis in the brain.

Given the successes observed in animal models of brain injury, major questions also now lie in the realm of immediate clinical application. Should new clinical trials be initiated to test the concept of ferroptosis inhibitors as key modulators of pathological cell death during brain injury? Could earlier trials be reinterpreted in the light of our new mechanistic understanding of this pathway? There is a long history of developing iron chelators and antioxidant small molecules as therapies for brain injury with varied success (reviewed in [117, $118,125-127])$. New approaches, incorporating our past knowledge of antioxidant function into our growing mechanistic knowledge of ferroptosis, may aid in the identification or development of compounds that lead to better ways of specifically blocking iron-dependent ROS accumulation in vivo. While many hurdles remain, the study of this process has the potential to lead to a new understanding of brain injury and new routes to effective treatment.

\section{Acknowledgement}

The authors thank members of the Dixon Lab for helpful discussions.

\section{Statement of Ethics}

The authors have no ethical conflicts to disclose.

\section{Disclosure Statement}

S.J.D. is on the scientific advisory board of Ferro Therapeutics. 


\section{Funding Sources}

This work was supported by an award from the NIH (1R01GM122923) to S.J.D.

\section{Author Contributions}

L.M. and S.J.D. wrote the manuscript.

\section{References}

1 Fuchs Y, Steller H. Programmed cell death in animal development and disease. Cell. 2011 Nov;147(4):742-58.

2 Galluzzi L, Vitale I, Aaronson SA, Abrams JM, Adam D, Agostinis P, et al. Molecular mechanisms of cell death: recommendations of the Nomenclature Committee on Cell Death 2018. Cell Death Differ. 2018 Mar; 25(3):486-541.

3 Yuan J, Kroemer G. Alternative cell death mechanisms in development and beyond. Genes Dev. 2010 Dec;24(23):2592-602.

4 Gudipaty SA, Conner CM, Rosenblatt J, Montell DJ. Unconventional Ways to Live and Die: Cell Death and Survival in Development, Homeostasis, and Disease. Annu Rev Cell Dev Biol. 2018 Oct;34(1):311-32.

5 Linkermann A, Stockwell BR, Krautwald S, Anders HJ. Regulated cell death and inflammation: an auto-amplification loop causes organ failure. Nat Rev Immunol. 2014 Nov; 14(11):759-67.

6 Vanden Berghe T, Linkermann A, JouanLanhouet S, Walczak H, Vandenabeele P. Regulated necrosis: the expanding network of non-apoptotic cell death pathways. Nat Rev Mol Cell Biol. 2014 Feb;15(2):135-47.

7 Ito Y, Ofengeim D, Najafov A, Das S, Saberi $\mathrm{S}$, Li Y, et al. RIPK1 mediates axonal degeneration by promoting inflammation and necroptosis in ALS. Science. 2016 Aug; 353(6299):603-8.

$8 \mathrm{Xu} \mathrm{D}$, Jin $\mathrm{T}$, Zhu $\mathrm{H}$, Chen $\mathrm{H}$, Ofengeim $\mathrm{D}$, Zou C, et al. TBK1 Suppresses RIPK1-Driven Apoptosis and Inflammation during Development and in Aging. Cell. 2018 Sep;174(6): 1477-1491.e19.

9 Andrabi SA, Kang HC, Haince JF, Lee YI, Zhang J, Chi Z, et al. Iduna protects the brain from glutamate excitotoxicity and stroke by interfering with poly(ADP-ribose) polymerinduced cell death. Nat Med. 2011 Jun;17(6): 692-9.

10 Eliasson MJ, Sampei K, Mandir AS, Hurn PD, Traystman RJ, Bao J, et al. Poly(ADP-ribose) polymerase gene disruption renders mice resistant to cerebral ischemia. Nat Med. 1997 Oct;3(10):1089-95.
11 Wang Y, An R, Umanah GK, Park H, Nambiar K, Eacker SM, et al. A nuclease that mediates cell death induced by DNA damage and poly(ADP-ribose) polymerase-1. Science. 2016 Oct;354(6308):aad6872-6872.

12 Dixon SJ, Lemberg KM, Lamprecht MR, Skouta R, Zaitsev EM, Gleason CE, et al. Ferroptosis: an iron-dependent form of nonapoptotic cell death. Cell. 2012 May;149(5):1060-72.

13 Stockwell BR, Friedmann Angeli JP, Bayir H, Bush AI, Conrad M, Dixon SJ, et al. Ferroptosis: A Regulated Cell Death Nexus Linking Metabolism, Redox Biology, and Disease. Cell. 2017 Oct;171(2):273-85.

14 Linkermann A, Skouta R, Himmerkus N, Mulay SR, Dewitz C, De Zen F, et al. Synchronized renal tubular cell death involves ferroptosis. Proc Natl Acad Sci USA. 2014 Nov;111(47): 16836-41.

15 Gao M, Monian P, Quadri N, Ramasamy R, Jiang X. Glutaminolysis and Transferrin Regulate Ferroptosis. Mol Cell. 2015 Jul;59(2):298-308.

16 Viswanathan VS, Ryan MJ, Dhruv HD, Gill S, Eichhoff OM, Seashore-Ludlow B, et al. Dependency of a therapy-resistant state of cancer cells on a lipid peroxidase pathway. Nature. 2017 Jul;547(7664):453-7.

17 Hangauer MJ, Viswanathan VS, Ryan MJ, Bole D, Eaton JK, Matov A, et al. Drug-tolerant persister cancer cells are vulnerable to GPX4 inhibition. Nature. 2017 Nov; 551(7679):247-50.

18 Friedmann Angeli JP, Schneider M, Proneth B, Tyurina YY, Tyurin VA, Hammond VJ, et al. Inactivation of the ferroptosis regulator Gpx4 triggers acute renal failure in mice. Nat Cell Biol. 2014 Dec;16(12):1180-91.

19 Ingold I, Berndt C, Schmitt S, Doll S, Poschmann G, Buday K, et al. Selenium Utilization by GPX4 Is Required to Prevent Hydroperoxide-Induced Ferroptosis. Cell. 2018 Jan; 172(3):409-422.e21.

20 Wenzel SE, Tyurina YY, Zhao J, St Croix CM, Dar HH, Mao G, et al. PEBP1 Wardens Ferroptosis by Enabling Lipoxygenase Generation of Lipid Death Signals. Cell. 2017 Oct;171(3):628641.e26.
21 Hirschhorn T, Stockwell BR. The development of the concept of ferroptosis. Free Radic Biol Med. 2018. DOI: 10.1016/j.freeradbiomed.2018.09.043.

22 Cao JY, Dixon SJ. Mechanisms of ferroptosis. Cell Mol Life Sci. 2016 Jun;73(11-12):2195209.

23 Vexler ZS, Wong A, Francisco C, Manabat C, Christen S, Täuber $M$, et al. Fructose-1,6bisphosphate preserves intracellular glutathione and protects cortical neurons against oxidative stress. Brain Res. 2003 Jan;960(1-2):90-8.

24 Lafemina MJ, Sheldon RA, Ferriero DM. Acute hypoxia-ischemia results in hydrogen peroxide accumulation in neonatal but not adult mouse brain. Pediatr Res. 2006 May;59(5):680-3.

25 Degterev A, Huang Z, Boyce M, Li Y, Jagtap $\mathrm{P}$, Mizushima N, et al. Chemical inhibitor of nonapoptotic cell death with therapeutic potential for ischemic brain injury. Nat Chem Biol. 2005 Jul;1(2):112-9.

26 Agmon E, Solon J, Bassereau P, Stockwell BR. Modeling the effects of lipid peroxidation during ferroptosis on membrane properties. Sci Rep. 2018 Mar;8(1):5155.

27 Yagoda N, von Rechenberg M, Zaganjor E, Bauer AJ, Yang WS, Fridman DJ, et al. RASRAF-MEK-dependent oxidative cell death involving voltage-dependent anion channels. Nature. 2007 Jun;447(7146):864-8.

28 Dolma S, Lessnick SL, Hahn WC, Stockwell BR. Identification of genotype-selective antitumor agents using synthetic lethal chemical screening in engineered human tumor cells. Cancer Cell. 2003 Mar;3(3):285-96.

29 Yang WS, Stockwell BR. Synthetic lethal screening identifies compounds activating iron-dependent, nonapoptotic cell death in oncogenic-RAS-harboring cancer cells. Chem Biol. 2008 Mar; 15(3):234-45.

30 Li Q, Han X, Lan X, Gao Y, Wan J, Durham $\mathrm{F}$, et al. Inhibition of neuronal ferroptosis protects hemorrhagic brain. JCI Insight. 2017 Apr;2(7):e90777.

31 Xie BS, Wang YQ, Lin Y, Mao Q, Feng JF, Gao GY, et al. Inhibition of ferroptosis attenuates tissue damage and improves long-term outcomes after traumatic brain injury in mice. CNS Neurosci Ther. 2018 Sep;33:1. 
32 Dixon SJ, Patel DN, Welsch M, Skouta R, Lee ED, Hayano M, et al. Pharmacological inhibition of cystine-glutamate exchange induces endoplasmic reticulum stress and ferroptosis. eLife. 2014 May;3:e02523.

33 Sato M, Kusumi R, Hamashima S, Kobayashi S, Sasaki S, Komiyama Y, et al. The ferroptosis inducer erastin irreversibly inhibits system $\mathrm{xc}-$ and synergizes with cisplatin to increase cisplatin's cytotoxicity in cancer cells. Sci Rep. 2018 Jan;8(1):968.

34 Bridges R, Lutgen V, Lobner D, Baker DA. Thinking outside the cleft to understand synaptic activity: contribution of the cystine-glutamate antiporter (System xc-) to normal and pathological glutamatergic signaling. Pharmacol Rev. 2012 Jul;64(3):780-802.

35 Yang WS, SriRamaratnam R, Welsch ME, Shimada K, Skouta R, Viswanathan VS, et al. Regulation of ferroptotic cancer cell death by GPX4. Cell. 2014 Jan;156(1-2):317-31.

36 Shimada K, Skouta R, Kaplan A, Yang WS, Hayano M, Dixon SJ, et al. Global survey of cell death mechanisms reveals metabolic regulation of ferroptosis. Nat Chem Biol. 2016 Jul;12(7):497-503.

37 Gaschler MM, Stockwell BR. Lipid peroxidation in cell death. Biochem Biophys Res Commun. 2017 Jan;482(3):419-25.

38 Skouta R, Dixon SJ, Wang J, Dunn DE, Orman M, Shimada K, et al. Ferrostatins inhibit oxidative lipid damage and cell death in diverse disease models. J Am Chem Soc. 2014 Mar;136(12):4551-6.

39 Kagan VE, Mao G, Qu F, Angeli JP, Doll S, Croix CS, et al. Oxidized arachidonic and adrenic PEs navigate cells to ferroptosis. Nat Chem Biol. 2017 Jan;13(1):81-90.

40 Doll S, Proneth B, Tyurina YY, Panzilius E, Kobayashi S, Ingold I, et al. ACSL4 dictates ferroptosis sensitivity by shaping cellular lipid composition. Nat Chem Biol. 2017 Jan;13(1): 91-8.

41 Dixon SJ, Winter GE, Musavi LS, Lee ED, Snijder B, Rebsamen M, et al. Human Haploid Cell Genetics Reveals Roles for Lipid Metabolism Genes in Nonapoptotic Cell Death. ACS Chem Biol. 2015 Jul;10(7):1604-9.

42 Yuan H, Li X, Zhang X, Kang R, Tang D. Identification of ACSL4 as a biomarker and contributor of ferroptosis. Biochem Biophys Res Commun. 2016 Sep;478(3):1338-43.

43 Alvarez SW, Sviderskiy VO, Terzi EM, Papagiannakopoulos T, Moreira AL, Adams S, et al. NFS1 undergoes positive selection in lung tumours and protects cells from ferroptosis. Nature. 2017 Nov;551(7682): 639-43.

44 Yang WS, Kim KJ, Gaschler MM, Patel M, Shchepinov MS, Stockwell BR. Peroxidation of polyunsaturated fatty acids by lipoxygenases drives ferroptosis. Proc Natl Acad Sci USA. 2016 Aug;113(34):E4966-75.

45 Li Y, Maher P, Schubert D. A role for 12-lipoxygenase in nerve cell death caused by glutathione depletion. Neuron. 1997 Aug;19(2): 453-63.
46 Karuppagounder SS, Alin L, Chen Y, Brand D, Bourassa MW, Dietrich K, et al. N-acetylcysteine targets 5 lipoxygenase-derived, toxic lipids and can synergize with prostaglandin E2 to inhibit ferroptosis and improve outcomes following hemorrhagic stroke in mice. Ann Neurol. 2018 Dec;84(6):854-72.

47 Shah R, Shchepinov MS, Pratt DA. Resolving the Role of Lipoxygenases in the Initiation and Execution of Ferroptosis. ACS Cent Sci. 2018 Mar;4(3):387-96.

48 Shah R, Margison K, Pratt DA. The Potency of Diarylamine Radical-Trapping Antioxidants as Inhibitors of Ferroptosis Underscores the Role of Autoxidation in the Mechanism of Cell Death. ACS Chem Biol. 2017 Oct; 12(10):2538-45.

49 Bannai S, Tsukeda H, Okumura H. Effect of antioxidants on cultured human diploid fibroblasts exposed to cystine-free medium. Biochem Biophys Res Commun. 1977 Feb; 74(4):1582-8

50 Murphy TH, Miyamoto M, Sastre A, Schnaar RL, Coyle JT. Glutamate toxicity in a neuronal cell line involves inhibition of cystine transport leading to oxidative stress. Neuron. 1989 Jun;2(6):1547-58.

51 Yonezawa M, Back SA, Gan X, Rosenberg PA, Volpe JJ. Cystine deprivation induces oligodendroglial death: rescue by free radical scavengers and by a diffusible glial factor. J Neurochem. 1996 Aug;67(2):566-73.

52 Oka A, Belliveau MJ, Rosenberg PA, Volpe JJ. Vulnerability of oligodendroglia to glutamate: pharmacology, mechanisms, and prevention. J Neurosci. 1993 Apr;13(4):1441-53.

53 Froissard P, Monrocq H, Duval D. Role of glutathione metabolism in the glutamate-induced programmed cell death of neuronallike PC12 cells. Eur J Pharmacol. 1997 May; 326(1):93-9.

54 Tan S, Wood M, Maher P. Oxidative stress induces a form of programmed cell death with characteristics of both apoptosis and necrosis in neuronal cells. J Neurochem. 1998 Jul; 71(1):95-105.

55 Davis JB, Maher P. Protein kinase C activation inhibits glutamate-induced cytotoxicity in a neuronal cell line. Brain Res. 1994 Jul; 652(1):169-73.

56 Tan S, Schubert D, Maher P. Oxytosis: A novel form of programmed cell death. Curr Top Med Chem. 2001 Dec;1(6):497-506.

57 Dixon SJ, Stockwell BR. The role of iron and reactive oxygen species in cell death. Nat Chem Biol. 2014 Jan;10(1):9-17.

58 Lewerenz J, Ates G, Methner A, Conrad M, Maher P. Oxytosis/Ferroptosis-(Re-) Emerging Roles for Oxidative Stress-Dependent Non-apoptotic Cell Death in Diseases of the Central Nervous System. Front Neurosci. 2018 Apr;12:214.

59 Gaschler MM, Hu F, Feng H, Linkermann A, Min W, Stockwell BR. Determination of the Subcellular Localization and Mechanism of Action of Ferrostatins in Suppressing Ferroptosis. ACS Chem Biol. 2018 Apr;13(4):1013-20.
60 Schnabel D, Salas-Vidal E, Narváez V, Sánchez-Carbente MR, Hernández-García D, Cuervo R, et al. Expression and regulation of antioxidant enzymes in the developing limb support a function of ROS in interdigital cell death. Dev Biol. 2006 Mar;291(2):291-9.

61 Chautan M, Chazal G, Cecconi F, Gruss P, Golstein P. Interdigital cell death can occur through a necrotic and caspase-independent pathway. Curr Biol. 1999 Sep;9(17):967-70.

62 Jiang L, Kon N, Li T, Wang SJ, Su T, Hibshoosh $\mathrm{H}$, et al. Ferroptosis as a p53-mediated activity during tumour suppression. Nature. 2015 Apr;520(7545):57-62.

63 Savaskan NE, Borchert A, Bräuer AU, Kuhn $\mathrm{H}$. Role for glutathione peroxidase-4 in brain development and neuronal apoptosis: specific induction of enzyme expression in reactive astrocytes following brain injury. Free Radic Biol Med. 2007 Jul;43(2):191-201.

64 Borchert A, Wang CC, Ufer C, Schiebel H, Savaskan NE, Kuhn H. The role of phospholipid hydroperoxide glutathione peroxidase isoforms in murine embryogenesis. J Biol Chem. 2006 Jul;281(28):19655-64.

65 Qian Z, Wilusz J. GRSF-1: a poly(A)+ mRNA binding protein which interacts with a conserved G-rich element. Nucleic Acids Res. 1994 Jun;22(12):2334-43.

66 Ufer C, Wang CC, Fähling M, Schiebel H, Thiele BJ, Billett EE, et al. Translational regulation of glutathione peroxidase 4 expression through guanine-rich sequence-binding factor 1 is essential for embryonic brain development. Genes Dev. 2008 Jul;22(13):1838-50.

67 Milasta S, Dillon CP, Sturm OE, Verbist KC, Brewer TL, Quarato G, et al. Apoptosis-Inducing-Factor-Dependent Mitochondrial Function Is Required for T Cell but Not B Cell Function. Immunity. 2016 Jan;44(1):88-102.

$68 \mathrm{Ke} \mathrm{FF}$, Vanyai HK, Cowan AD, Delbridge AR, Whitehead L, Grabow S, et al. Embryogenesis and Adult Life in the Absence of Intrinsic Apoptosis Effectors BAX, BAK, and BOK. Cell. 2018 May;173(5):1217-1230.e17.

69 Yant LJ, Ran Q, Rao L, Van Remmen H, Shibatani T, Belter JG, et al. The selenoprotein GPX4 is essential for mouse development and protects from radiation and oxidative damage insults. Free Radic Biol Med. 2003 Feb;34(4): 496-502.

70 Sengupta A, Lichti UF, Carlson BA, Cataisson C, Ryscavage AO, Mikulec C, et al. Targeted disruption of glutathione peroxidase 4 in mouse skin epithelial cells impairs postnatal hair follicle morphogenesis that is partially rescued through inhibition of COX-2.J Invest Dermatol. 2013 Jul;133(7):1731-41.

71 Yoo SE, Chen L, Na R, Liu Y, Rios C, Van Remmen $\mathrm{H}$, et al. Gpx4 ablation in adult mice results in a lethal phenotype accompanied by neuronal loss in brain. Free Radic Biol Med. 2012 May;52(9):1820-7.

72 Sato H, Shiiya A, Kimata M, Maebara K, Tamba $\mathrm{M}$, Sakakura $\mathrm{Y}$, et al. Redox imbalance in cystine/glutamate transporter-deficient mice. J Biol Chem. 2005 Nov;280(45):37423-9. 
73 Chintala S, Li W, Lamoreux ML, Ito S, Wakamatsu K, Sviderskaya EV, et al. Slc7a11 gene controls production of pheomelanin pigment and proliferation of cultured cells. Proc Natl Acad Sci USA. 2005 Aug;102(31): 10964-9.

74 McCullagh EA, Featherstone DE. Behavioral characterization of system xc- mutant mice. Behav Brain Res. 2014 May;265:1-11.

75 Prigge JR, Coppo L, Martin SS, Ogata F, Miller CG, Bruschwein MD, et al. Hepatocyte Hyperproliferation upon Liver-Specific Co-disruption of Thioredoxin-1, Thioredoxin Reductase-1, and Glutathione Reductase. Cell Reports. 2017 Jun;19(13):2771-81.

76 Sheldon RA, Jiang X, Francisco C, Christen S, Vexler ZS, Täuber MG, et al. Manipulation of antioxidant pathways in neonatal murine brain. Pediatr Res. 2004 Oct;56(4):656-62.

77 Fan P, Yamauchi T, Noble LJ, Ferriero DM. Age-dependent differences in glutathione peroxidase activity after traumatic brain injury. J Neurotrauma. 2003 May;20(5):437-45.

78 Do Van B, Gouel F, Jonneaux A, Timmerman K, Gelé P, Pétrault M, et al. Ferroptosis, a newly characterized form of cell death in Parkinson's disease that is regulated by PKC. Neurobiol Dis. 2016 Oct;94:169-78.

79 Back SA, Luo NL, Mallinson RA, O’Malley JP, Wallen LD, Frei B, et al. Selective vulnerability of preterm white matter to oxidative damage defined by F2-isoprostanes. Ann Neurol. 2005 Jul;58(1):108-20.

80 Dommergues MA, Gallego J, Evrard P, Gressens $P$. Iron supplementation aggravates periventricular cystic white matter lesions in newborn mice. Eur J Paediatr Neurol. 1998;2(6): 313-8.

81 Brault S, Martinez-Bermudez AK, Roberts J 2nd, Cui QL, Fragoso G, Hemdan S, et al. Cytotoxicity of the $\mathrm{E}(2)$-isoprostane $15-\mathrm{E}(2 \mathrm{t})$ Iso $\mathrm{P}$ on oligodendrocyte progenitors. Free Radic Biol Med. 2004 Aug;37(3):358-66.

82 Novgorodov SA, Voltin JR, Gooz MA, Li L, Lemasters JJ, Gudz TI. Acid sphingomyelinase promotes mitochondrial dysfunction due to glutamate-induced regulated necrosis. J Lipid Res. 2018 Feb;59(2):312-29.

83 Tuo QZ, Lei P, Jackman KA, Li XL, Xiong H, Li XL, et al. Tau-mediated iron export prevents ferroptotic damage after ischemic stroke. Mol Psychiatry. 2017 Nov;22(11): 1520-30.

84 DeGregorio-Rocasolano N, Martí-Sistac O, Ponce J, Castelló-Ruiz M, Millán M, Guirao $\mathrm{V}$, et al. Iron-loaded transferrin (Tf) is detrimental whereas iron-free $\mathrm{Tf}$ confers protection against brain ischemia by modifying blood Tf saturation and subsequent neuronal damage. Redox Biol. 2018 May;15:14358.

85 Zille M, Karuppagounder SS, Chen Y, Gough PJ, Bertin J, Finger J, et al. Neuronal Death After Hemorrhagic Stroke In Vitro and In Vivo Shares Features of Ferroptosis and Necroptosis. Stroke. 2017 Apr;48(4):1033-43.
86 Zhang Z, Wu Y, Yuan S, Zhang P, Zhang J, Li $\mathrm{H}$, et al. Glutathione peroxidase 4 participates in secondary brain injury through mediating ferroptosis in a rat model of intracerebral hemorrhage. Brain Res. 2018 Dec;1701:112-25.

87 Zhao X, Ting SM, Liu CH, Sun G, Kruzel M, Roy-O'Reilly M, et al. Neutrophil polarization by IL-27 as a therapeutic target for intracerebral hemorrhage. Nat Commun. 2017 Sep;8(1):602.

88 Roth TL, Nayak D, Atanasijevic T, Koretsky AP, Latour LL, McGavern DB. Transcranial amelioration of inflammation and cell death after brain injury. Nature. 2014 Jan;505(7482): 223-8.

89 Stoica BA, Faden AI. Cell death mechanisms and modulation in traumatic brain injury. Neurotherapeutics. 2010 Jan;7(1):3-12.

90 Raghupathi R. Cell death mechanisms following traumatic brain injury. Brain Pathol. 2004 Apr;14(2):215-22.

91 Li Q, Weiland A, Chen X, Lan X, Han X, Durham F, et al. Ultrastructural Characteristics of Neuronal Death and White Matter Injury in Mouse Brain Tissues After Intracerebral Hemorrhage: Coexistence of Ferroptosis, Autophagy, and Necrosis. Front Neurol. 2018 Jul;9:581.

92 Palmer C, Menzies SL, Roberts RL, Pavlick G, Connor JR. Changes in iron histochemistry after hypoxic-ischemic brain injury in the neonatal rat. J Neurosci Res. 1999 Apr;56(1): 60-71.

93 Palmer C, Roberts RL, Bero C. Deferoxamine posttreatment reduces ischemic brain injury in neonatal rats. Stroke. 1994 May;25(5): 1039-45.

94 Papazisis G, Pourzitaki C, Sardeli C, Lallas A, Amaniti E, Kouvelas D. Deferoxamine decreases the excitatory amino acid levels and improves the histological outcome in the hippocampus of neonatal rats after hypoxiaischemia. Pharmacol Res. 2008 Jan;57(1): 73-8.

95 Bayir H, Kagan VE, Tyurina YY, Tyurin V, Ruppel RA, Adelson PD, et al. Assessment of antioxidant reserves and oxidative stress in cerebrospinal fluid after severe traumatic brain injury in infants and children. Pediatr Res. 2002 May;51(5):571-8.

96 Namura S, Iihara K, Takami S, Nagata I, Kikuchi $\mathrm{H}$, Matsushita K, et al. Intravenous administration of MEK inhibitor U0126 affords brain protection against forebrain ischemia and focal cerebral ischemia. Proc Natl Acad Sci USA. 2001 Sep;98(20):11569-74.

97 Satoh T, Nakatsuka D, Watanabe Y, Nagata I, Kikuchi H, Namura S. Neuroprotection by MAPK/ERK kinase inhibition with U0126 against oxidative stress in a mouse neuronal cell line and rat primary cultured cortical neurons. Neurosci Lett. 2000 Jul;288(2):163-6.

$98 \mathrm{Xu}$ X, Chua CC, Kong J, Kostrzewa RM, Kumaraguru U, Hamdy RC, et al. Necrostatin-1 protects against glutamate-induced glutathione depletion and caspase-independent cell death in HT-22 cells. J Neurochem. 2007 Dec; 103(5):2004-14.
99 Takahashi N, Duprez L, Grootjans S, Cauwels A, Nerinckx W, DuHadaway JB, et al. Necrostatin-1 analogues: critical issues on the specificity, activity and in vivo use in experimental disease models. Cell Death Dis. 2012 Nov;3(11):e437-437.

$100 \mathrm{Xu}$ X, Chua KW, Chua CC, Liu CF, Hamdy $\mathrm{RC}$, Chua BH. Synergistic protective effects of humanin and necrostatin-1 on hypoxia and ischemia/reperfusion injury. Brain Res. 2010 Oct;1355:189-94.

101 Rouault TA. Iron metabolism in the CNS implications for neurodegenerative diseases. Nat Rev Neurosci. 2013 Aug;14(8):551-64.

102 Masaldan S, Bush AI, Devos D, Rolland AS, Moreau C. Striking while the iron is hot: iron metabolism and ferroptosis in neurodegeneration. Free Radic Biol Med. 2018. DOI:10.1016/j.freeradbiomed.2018.09.033.

103 Gouel F, Do Van B, Chou ML, Jonneaux A, Moreau C, Bordet R, et al. The protective effect of human platelet lysate in models of neurodegenerative disease: involvement of the Akt and MEK pathways. J Tissue Eng Regen Med. 2017 Nov;11(11):3236-40.

104 Lane DJ, Ayton S, Bush AI. Iron and Alzheimer's Disease: An Update on Emerging Mechanisms. J Alzheimers Dis. 2018;64 s1:S379-95.

105 Zhang YH, Wang DW, Xu SF, Zhang S, Fan YG, Yang YY, et al. $\alpha$-Lipoic acid improves abnormal behavior by mitigation of oxidative stress, inflammation, ferroptosis, and tauopathy in P301S Tau transgenic mice. Redox Biol. 2018 Apr;14:535-48.

106 Nasoohi S, Simani L, Khodagholi F, Nikseresht S, Faizi M, Naderi N. Coenzyme Q10 supplementation improves acute outcomes of stroke in rats pretreated with atorvastatin. Nutr Neurosci. 2017 Sep;23: 1-9.

107 Vande Voorde J, Ackermann T, Pfetzer N, Sumpton D, Mackay G, Kalna G, et al. Improving the metabolic fidelity of cancer models with a physiological cell culture medium. Sci Adv. 2019 Jan;5:eaau7314.

108 Cheah JH, Kim SF, Hester LD, Clancy KW, Patterson SE 3rd, Papadopoulos V, et al NMDA receptor-nitric oxide transmission mediates neuronal iron homeostasis via the GTPase Dexras1. Neuron. 2006 Aug;51(4): 431-40.

109 Xu H, Liu X, Xia J, Yu T, Qu Y, Jiang H, et al. Activation of NMDA receptors mediated iron accumulation via modulating iron transporters in Parkinson's disease. FASEB J. 2018. DOI: 10.1096/fj.201800060RR.

$110 \mathrm{Xu} \mathrm{H}$, Jiang H, Xie J. New Insights into the Crosstalk between NMDARs and Iron: Implications for Understanding Pathology of Neurological Diseases. Front Mol Neurosci. 2017 Mar; 10:71.

111 Chaitanya GV, Steven AJ, Babu PP. PARP-1 cleavage fragments: signatures of cell-death proteases in neurodegeneration. Cell Commun Signal. 2010 Dec;8(1):31. 
112 Wang H, Sun L, Su L, Rizo J, Liu L, Wang LF, et al. Mixed lineage kinase domain-like protein MLKL causes necrotic membrane disruption upon phosphorylation by RIP3. Mol Cell. 2014 Apr;54(1):133-46.

113 Oh-Hashi K, Nomura Y, Shimada K, Koga H, Hirata Y, Kiuchi K. Transcriptional and post-translational regulation of mouse cation transport regulator homolog 1 . Mol Cell Biochem. 2013 Aug;380(1-2):97-106.

114 Mungrue IN, Pagnon J, Kohannim O, Gargalovic PS, Lusis AJ. CHAC1/MGC4504 is a novel proapoptotic component of the unfolded protein response, downstream of the ATF4-ATF3-CHOP cascade. J Immunol. 2009 Jan;182(1):466-76.

115 Ji J, Kline AE, Amoscato A, Samhan-Arias AK, Sparvero LJ, Tyurin VA, et al. Lipidomics identifies cardiolipin oxidation as a mitochondrial target for redox therapy of brain injury. Nat Neurosci. 2012 Oct; 15(10):1407-13.

116 Wang $\mathrm{H}$, Zhang R, Bridle KR, Jayachandran A, Thomas JA, Zhang W, et al. Two-photon dual imaging platform for in vivo monitoring cellular oxidative stress in liver injury. Sci Rep. 2017 Mar;7(1):45374.

117 Hall ED, Vaishnav RA, Mustafa AG. Antioxidant therapies for traumatic brain injury. Neurotherapeutics. 2010 Jan;7(1):51-61.
118 Hall ED, Wang JA, Miller DM, Cebak JE, Hill RL. Newer pharmacological approaches for antioxidant neuroprotection in traumatic brain injury. Neuropharmacology. 2019 Feb;145(Pt B):247-58.

119 Kim SE, Zhang L, Ma K, Riegman M, Chen F, Ingold I, et al. Ultrasmall nanoparticles induce ferroptosis in nutrient-deprived cancer cells and suppress tumour growth. Nat Nanotechnol. 2016 Nov;11(11):977-85.

120 Zhang Y, Sun C, Zhao C, Hao J, Zhang Y, Fan B, et al. Ferroptosis Inhibitor SRS 16-86 Attenuates Ferroptosis and Promotes Functional Recovery in Contusion Spinal Cord Injury. Brain Res. 2018. DOI: 10.1016/j. brainres.2018.10.023.

121 Kim JH, Lewin TM, Coleman RA. Expression and characterization of recombinant rat Acyl-CoA synthetases 1, 4, and 5. Selective inhibition by triacsin $\mathrm{C}$ and thiazolidinediones. J Biol Chem. 2001 Jul;276(27):2466773.

122 Cheng P, Wang J, Shao W. Monounsaturated Fatty Acid Intake and Stroke Risk: A Meta-analysis of Prospective Cohort Studies. J Stroke Cerebrovasc Dis. 2016 Jun;25(6): 1326-34.
123 Moreau C, Duce JA, Rascol O, Devedjian JC, Berg D, Dexter D, et al.; FAIRPARK-II study group. Iron as a therapeutic target for Parkinson's disease. Mov Disord. 2018 Apr; 33(4):568-74.

124 Zecca L, Youdim MB, Riederer P, Connor JR, Crichton RR. Iron, brain ageing and neurodegenerative disorders. Nat Rev Neurosci. 2004 Nov;5(11):863-73.

125 Fernández-Gajardo R, Matamala JM, Carrasco R, Gutiérrez R, Melo R, Rodrigo $R$. Novel therapeutic strategies for traumatic brain injury: acute antioxidant reinforcement. CNS Drugs. 2014 Mar;28(3): $229-48$.

126 Cui HJ, He HY, Yang AL, Zhou HJ, Wang C, Luo JK, et al. Efficacy of deferoxamine in animal models of intracerebral hemorrhage: a systematic review and stratified meta-analysis. PLoS One. 2015 May;10(5):e0127256.

127 Warkentin LM, Auriat AM, Wowk S, Colbourne F. Failure of deferoxamine, an iron chelator, to improve outcome after collagenase-induced intracerebral hemorrhage in rats. Brain Res. 2010 Jan;1309:95-103.

128 Magtanong L, Ko PJ, To M, Cao JY, Forcina GC, Tarangelo A, et al. Exogenous Monounsaturated Fatty Acids Promote a Ferroptosis-Resistant Cell State. Cell Chem Biol. 2018 Dec 11. pii: S2451-9456(18)30438-0. 